\title{
Drug Repurposing: In Silico Modeling of Streptococcus Infection
}

\author{
Ranajit Nath ${ }^{1}$, Ratna Roy ${ }^{2}$, Soubhik bhattacharyya ${ }^{3}$, Sourav Datta ${ }^{4}$ \\ ${ }^{1}$ Department of Pharmaceutics, NSHM Knowledge Campus, Kolkata- Group of Institutions, Kolkata, West \\ Bengal, India. \\ ${ }^{2}$ Department of Pharmacology, NSHM Knowledge Campus, Kolkata- Group of Institutions, Kolkata, West \\ Bengal, India \\ ${ }^{3}$ Department of Pharmaceutics, NSHM knowledge campus, Kolkata- Group of institutions. \\ Kolkata, West Bengal, India. \\ ${ }^{4}$ Department of Pharmaceutics, NSHM Knowledge Campus, Kolkata- Group of Institutions, Kolkata, West \\ Bengal, India
}

\begin{abstract}
Article Info

Volume 8, Issue 3

Page Number : 907-919

\section{Publication Issue}

May-June-2021

\section{Article History}

Accepted : 18 June 2021

Published : 25 June 2021

Occasionally there are explosive outbreaks of infectious diseases worldwide and they occur without any immediate epidemiological or microbiological explanations. Some of the bacterial infections that are often considered to be epidemic-prone are plague, cholera and Streptococcus infections. Continuous research works are done in search for potential medications that could increase the medical arsenal against these types of diseases. Our present work is focused upon repurposing the drugs: ciprofloxacin, norfloxacin, moxifloxacin, diltiazem against Streptococcus infections in blood, on skin, and in throat. We are hopeful that our finding will enrich the rational drug design against Streptococcus infections.

Keywords : Streptococcus infection, docking, protein data bank, ADME, ligands
\end{abstract}

\section{INTRODUCTION}

One of the major bacterial infections that are deemed to epidemic-prone worldwide is Streptococcus infection.

Streptococci are gram positive, sphere shaped bacteria and one of the several species of Streptococcus is usually the cause of Streptococcus infections. These infections usually affect the throat, skin and bloodstream $^{[1]}$.

Streptococcal infection in the throat, usually known as pharyngitis or strep throat, is usually found to effect children of 5-15 years age group. The symptoms usually include throat becoming sore including chills, fever, headache, nausea, vomiting and a general feeling of illness. The throat becomes beefy red while the tonsils get swollen with or without patches of pus $^{[2]}$.

Some of the skin diseases caused due to direct infection with streptococcus are impetigo, ecthyma, and cellulitis, erysipelas, necrotizing fasciitis and Streptococcal perianal and/or vulvar dermatitis. Besides these, Streptococci are capable of causing skin 
disease through indirect infection of the skin such as psoriasis and scarlet fever ${ }^{[3]}$.

Although streptococcus don't normally live in the blood stream but they are capable of invading into our blood which is known as epticemia. This occurs when bacterial infection bacterial infection from elsewhere in the body enters the bloodstream. This condition is very dangerous as the bacteria and their toxins maybe possibly be carried by blood and circulated into the entire body. If during such condition the streptococcal bacteria releases toxins in multiple organs, another rare, life threatening condition called streptococcal toxic shock syndrome may emerge, which can cause organ failure and death $^{[4]}$.

\section{$5 \mathrm{LI}^{[5]}$}

The phi812 virion contains an isometric head $90 \mathrm{~nm}$ in diameter and a long contractile tail about $240 \mathrm{~nm}$ ending with a baseplate. These are dynamic at native phages. phi812 baseplate proteins rearranged into two layers parallel to the bacterial cell wall upon binding to S. aureus, The double-layered baseplate architecture, which is specific for the contracted tail, is structurally distinct and has been observed in negative-stain micrographs of many Myoviridae phages. A unique characteristic feature of the doublelayered baseplate is compact building blocks which are mentioned as thick legs of a "Victorian side table," organized in two concentrical hexamers. Each of the legs of phi8112 is a complex of a trimer of receptorbinding proteins \& a trimer of elongated "tripod" proteins. The two trimers have a common threefold axis by forming a cone-shaped "receptor-tripod complex". The tripod proteins contain the narrow tip of the cone and three elongated protrusions which form peripheral regions of the receptor-tripod complex base.

\section{$4 \mathrm{PQL} \mathrm{L}^{[6]}$}

The RepA NTD has an overall fold composed of a central winged HTH flanked by an N-terminal helixstrand-helix and C-terminal helix-loop motif, with overall topology [ $\alpha 1$ (residues $8-14)-\beta 1(16-20)-\alpha 2(21-$
27)- $\alpha 3(33-53)-\quad \alpha 4(67-76)-\alpha 5(79-94)-\beta 2(94-100)-$ $\beta 3(106-112)-\alpha 6(116-129)]$. The helix-strand-helix \& C-terminal helix function to create an extensive dimer-of-dimers or tetramer. For the formation of the dimers, the $\beta 1$ takes strand from two NTDs interdigitate via antiparallel interactions. The dimer is again stabilized by multiple contacts between residues in helices $\alpha 1$ and $\alpha 6$. The final resultant dimer interface buries 2,005 $⿱ 2$ 2 of protein surface from solvent. By the orthogonal packing of two NTD dimers, the RepA tetramer is created. Formation of the compact tetramer is the deep insertion of the Nterminal tails of each subunit into complementary hydrophobic cavities located at the junction of $\beta 2-\beta 3$ and $\alpha 3$. The residues mediating oligomerization are highly conserved. However, tetramer formation buries 6,865 22 of surface area by supporting the notion that RepA is tetrameric.

\section{PT7 ${ }^{[6,7]}$}

These proteins are organized in three main regions: an N-terminal domain (NTD) consisting of $\sim 120$, a long and variable linker region ( $\sim 30-50$ residues), and a C-terminal domain (CTD) of $\sim 120$ residues. For replication purpose, the NTD and CTD are both important. The NTD shows the highest level of sequence conservation, that have resulted in the designation of plasmids that encode these proteins as the RepA N replicon family. However, RepA CTD regions exhibit homology between plasmids found out in genus-specific clusters, suggesting that this domain may perform a host-specific role. Moreover, the function of the RepA CTD stays enigmatic, recent studies have indicated that the NTD mediates DNA binding and interacts with iterons that reside within the plasmid origin. The essential roles are played by RepA proteins in multi-resistance plasmid retention marks them as attractive targets for the development of specific chemotherapeutics. Nevertheless, the successful design of such compounds necessitates structural and mechanistic insight. 


\section{Ciprofloxacin $^{[8]}$}

Ciprofloxacin is an antibiotic drug falling under fluoroquinolones derivatives, which are mainly used in the treatment of mild to moderate urinary and respiratory infections caused by vulnerable microorganisms. Ciprofloxacin is a second generation, synthetic and broad-spectrum antibiotic. Chemical structure of ciprofloxacin is composed of the main quinolone ring which is basically quinoline- $4(1 \mathrm{H})$ one having functional groups like cyclopropyl, carboxylic acid, flouro and piperazin-1-yl substituents at the positions 1, 3, 6 and 7 respectively. It also acts as an antimicrobial agent, an environmental contaminant and a xenobiotic.

\section{Norfloxacin $^{[9,10]}$}

Norfloxacin is a basically quinoline monocarboxylic acid with an aryl piperazine as substituents. It is another antibiotic drug classified in fluoroquinolone derivates. It is a first generation, synthetic and broadspectrum antibiotic. Norfloxacin is a basically quinoline monocarboxylic acid with an aryl piperazine as substituents. It is used in the treatment of urinary tract infections and protasis.

\section{Moxifloxacin $^{[11]}$}

Moxifloxacin is a new drug moiety categorized under fluoroquinolone derivates. Moxifloxacin is fourth generation synthetic antibiotic and also used as an antibacterial agent. Chemical structure of moxifloxacin is composed of the main quinolone ring with the functional groups cyclopropyl, flouro, (4aS,7aS)-octahydro-6H-pyrrolo[3,4-b] pyridine-6-yl, methoxy substituents at positions 1,6,7,8 respectively. It is used for pneumonias, bronchitis, sinusitis.

\section{Diltiazem $^{[12]}$}

Diltiazem is a drug classified under benzothiazepine derivative. Diltiazem is categorized under a firstgeneration calcium channel blocker also used in the treatment of hypertension and angina pectoris. Diltiazem has antiarrhythmic properties.

\section{METHODS AND MATERIAL}

\section{Procedure:}

\section{1. ligand Screening}

For the initial Ligand screening purposes, a web-based tool named SwissADME (https://www.swiss adme.ch/) was used to eliminate a few compounds according to Lipinski's rule of five parameters ${ }^{[13]}$. For a compound to qualify as ligand it should Have $<500$ Da molecular weight, a high lipophilicity i.e. value of Log $\mathrm{P}$ being less than 5, hydrogen bond acceptors being less than 10 and $\mathrm{H}$-bond donors less than 5 . Any compound with more than 2 violations was ruled out for further study (Lipinski2004).

\section{Protein Preparation and Active site Determination.}

Required protein in pdb format was downloaded from the website rcsb.org, commonly known as the Protein Data Bank ${ }^{[14,15]}$. 3D conformers of the ligand were downloaded from PubChem. Using PyMOL (Version 2.4.1) software water molecules as well as native ligands from the protein were removed, defined as cleaning/purification of the protein for further application. Using a web server called Deep Site Active Pockets of the proteins were calculated ${ }^{[16]}$. The results calculated by the web server were in the form of different ids, centres and scores. Scoring In deep site was using neural networking based on following instructions using DCNN

architecture.

https://academic.oup.com/bioinformatics/article/33/19 /3036/3859178 Center values for the grid were selected keeping score greater than 0.98 .

UCSF Chimera (Version 1.14) was used to prepare the receptor using Dock Prep function.

Dock Prep prepared structures for Docking using these functions:

- deleting water molecules

- repairing truncated sidechains 
- adding hydrogens

- assigning partial charges

- writing files in Mol2 format

1. In silico Docking Using Auto Dock Vina

Auto dock Vina (Version 1.1.2) along with UCSF Chimera (Version 1.14) was used for molecular Docking Studies ${ }^{[17,18]}$. Centre values and size of the grid of different scores were used from DEEPSITE calculations done above.

Following Parameters were set in auto dock vina.

\section{Receptor options -}

- Add hydrogens in Chimera (true/false) - whether to add hydrogens in Chimera before calling the script. The receptor prep script will check for hydrogens and add them if they are missing. Auto Dock Vina needs the polar (potentially H-bonding) hydrogens to identify atom types for scoring purposes.

- Merge charges and remove non-polar hydrogens (true/false) - note Auto Dock Vina does not use charges or nonpolar hydrogens, so this setting is not expected to affect results except for the presence or absence of nonpolar hydrogens in the processed receptor.

- Merge charges and remove lone pairs (true/false) note Auto Dock Vina does not use charges or lone pairs, so this setting is not expected to affect results except for the presence or absence of lone pairs in the processed receptor (and there may not have been any lone pairs to start with)

\section{- Ignore waters (true/false)}

- Ignore chains of non-standard residues (true/false) ignore chains composed entirely of residues other than the 20 standard amino acids.

- Ignore all non-standard residues (true/false) ignore all residues other than the 20 standard amino acids.

\section{For Ligands}

- Merge charges and remove non-polar hydrogens (true/false) - note Auto Dock Vina does not use charges or nonpolar hydrogens, so this setting is not expected to affect results except for the presence or absence of nonpolar hydrogens in the ligand output files

- Merge charges and remove lone pairs (true/false) note Auto Dock Vina does not use charges or lone pairs, so this setting is not expected to affect results except for the presence or absence of lone pairs in the ligand output files (and there may not have been any lone pairs to start with)

\section{Docking parameters}

- Number of binding modes (1-10, 10) - maximum number of binding modes to generate

- Exhaustiveness of search $(1-8,8)$ - thoroughness of search, roughly proportional to time

- Maximum energy difference (kcal/mol) (1-3,3) maximum score range; binding

modes with scores not within this range of the best score will be discarded. The docking results were calculated by Auto dock vina using it's Scoring function and results were displayed in the form of Scores and RMSD values. Docking results with the highest value score accompanied by negative sign and least RMSD values were chosen for further studies.

\section{Residue Analysis}

PyMOL was used for visualization of interactions of the docked structure at the ligand sites ${ }^{[19]}$.

Discovery Studio 2020 was used to study the ligand interactions and total number of

residues $^{[20]}$. It was also used to plot the 2D structure of the interactions and residues.

5.Statistical Analysis: Descriptive, estimation and Hypothesis testing with confidence interval of $95 \%$ was applied to data using formula 1 given below ${ }^{[21]}$.

$$
C I=\bar{x} \pm z \frac{s}{\sqrt{n}}
$$




\section{RESULTS AND DISCUSSION}

\section{Bioavailability Radar:}

For the further analysis on four selected ligands viz

Norfloxacin, Ciprofloxacin, Moxifloxacin and
Diltiazem a more demonstrating and comprehensive study was performed using bioavailability radar. Bioavailability radar is elucidated tool used for investigation of the drug likeness of the ligands based on eight parameters.

Table 1 : shows the bioavailability radar charts of selected ligands

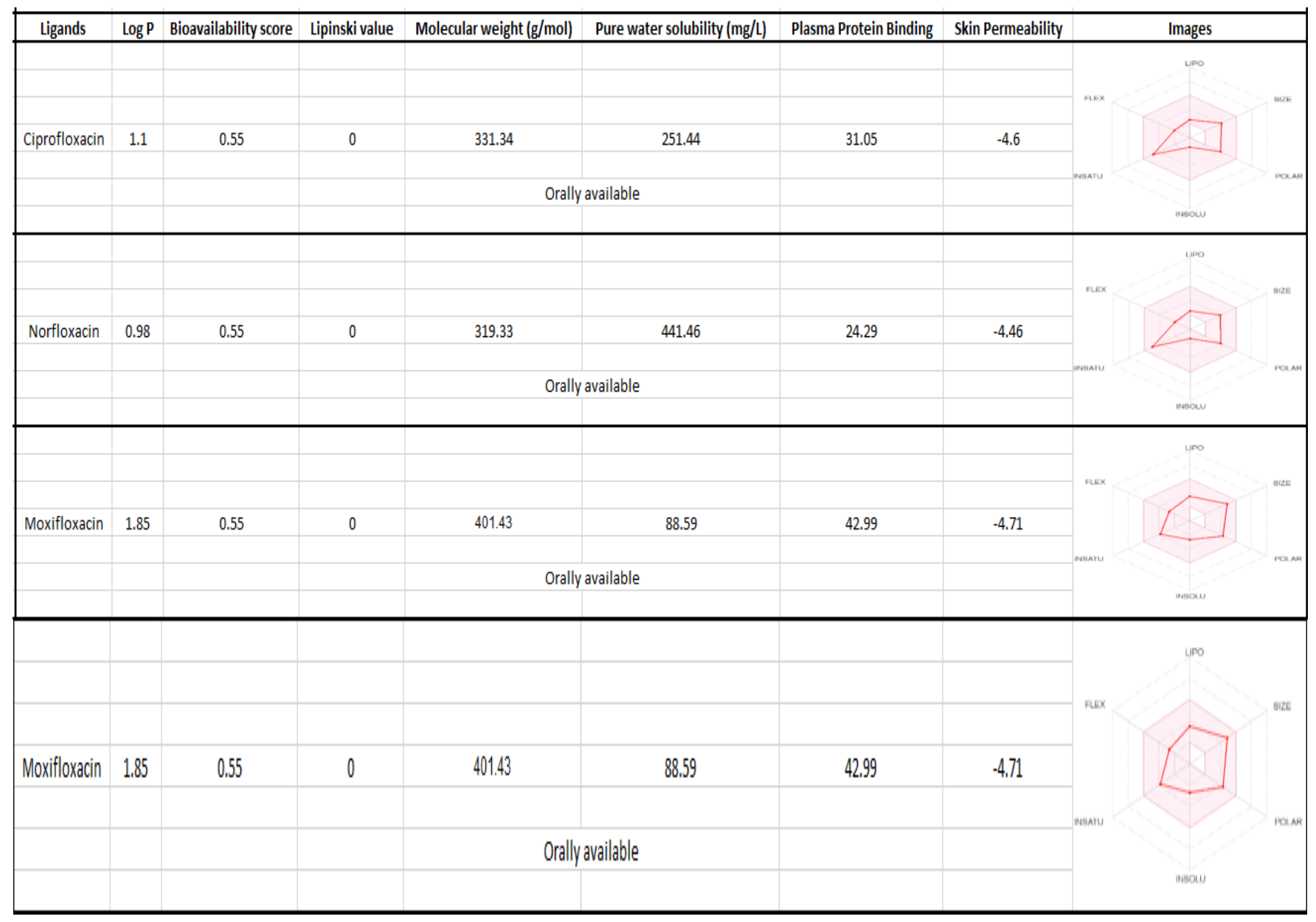

\section{Molecular Docking}

The docking result was collected from Auto-Dock vina in the form of Dock score for all the three proteins docked with above mentioned four ligands, average docking Score of each ligand accumulated to average dock score of three proteins were taken. Standard deviation and Confidence interval were reckoned, depending on the confidence interval minimum value of dock score for each ligand was reckoned. All the dock scores above the minimum score could be considered for further evaluations. 
Fig 1: Shows the average aggregate dock score for 3 proteins with respective ligands

Average Dock score vs. Ligands

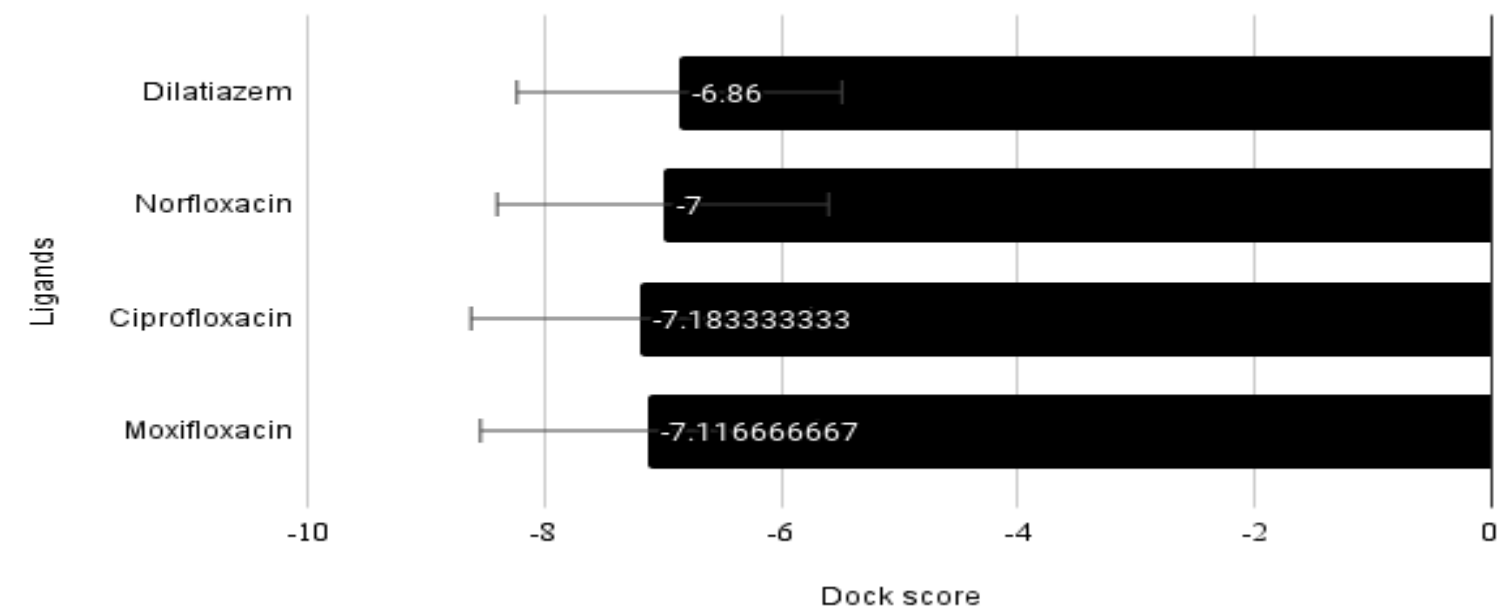

Table 2: Shows the standard deviation, confidence interval at $95 \%$ with minimum dock score

\begin{tabular}{|c|c|c|c|c|c|}
\hline Ligands & Average & $\begin{array}{c}\text { Standard } \\
\text { deviation }\end{array}$ & $\begin{array}{c}\text { Sample } \\
\text { size }\end{array}$ & $\begin{array}{c}\text { Confidence } \\
\text { Interval 95\% }\end{array}$ & $\begin{array}{c}\text { Min Score in } \\
\text { 95\% } \\
\text { confidence }\end{array}$ \\
\hline Diltiazem & -6.933333333 & 0.61210021 & 6 & 0.642359775 & -6.290973558 \\
\hline Norfloxacin & -7.1 & 0.715541753 & 6 & 0.750915017 & -6.349084983 \\
\hline ciprofloxacin & -7.05 & 0.918150314 & 6 & 0.963539663 & -6.086460337 \\
\hline Moxifloxacin & -7.116666667 & 0.204124145 & 6 & 0.214215153 & -6.902451514 \\
\hline
\end{tabular}

Fig 2: shows selected max docking score with -ve sign of all the ligands with all the proteins considered in studies

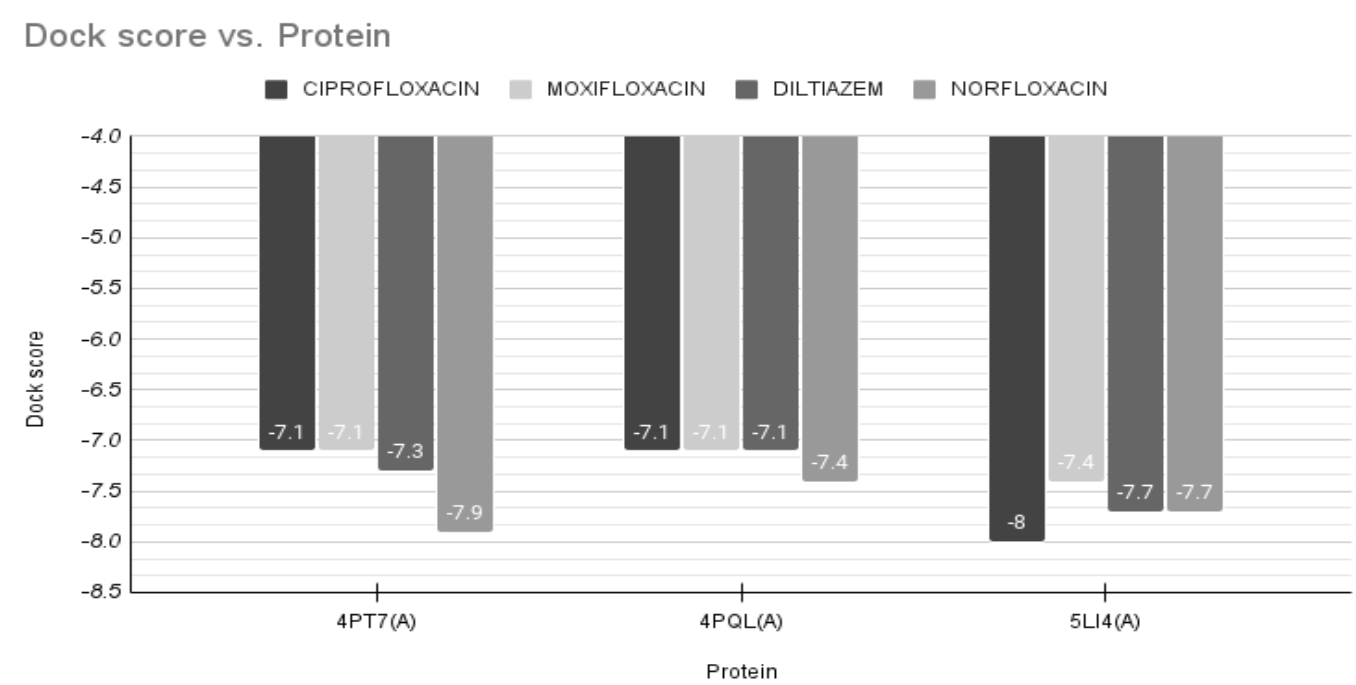


Table 3: showing interactions and max docking score with -ve sign of Norfloxacin and Diltiazem with protein used in study.

\begin{tabular}{|c|c|c|c|}
\hline Dock scores & Ligands & Proteins & Amino acid interactions \\
\hline-7.1 & Dilatiazam & 4PQL(A) & 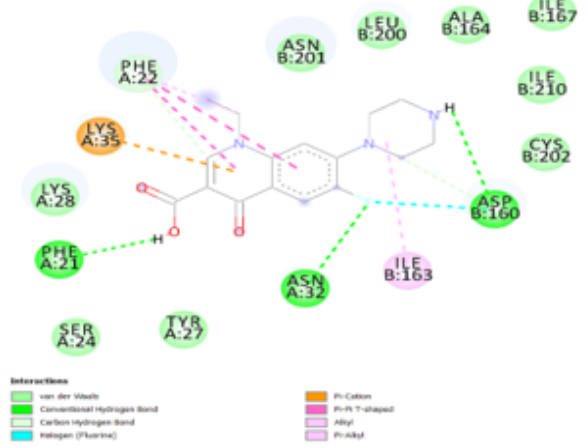 \\
\hline-6.9 & Dilatiazam & $4 P Q L(A)$ & 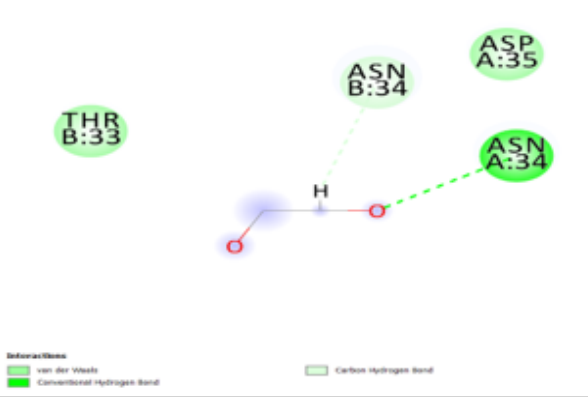 \\
\hline
\end{tabular}

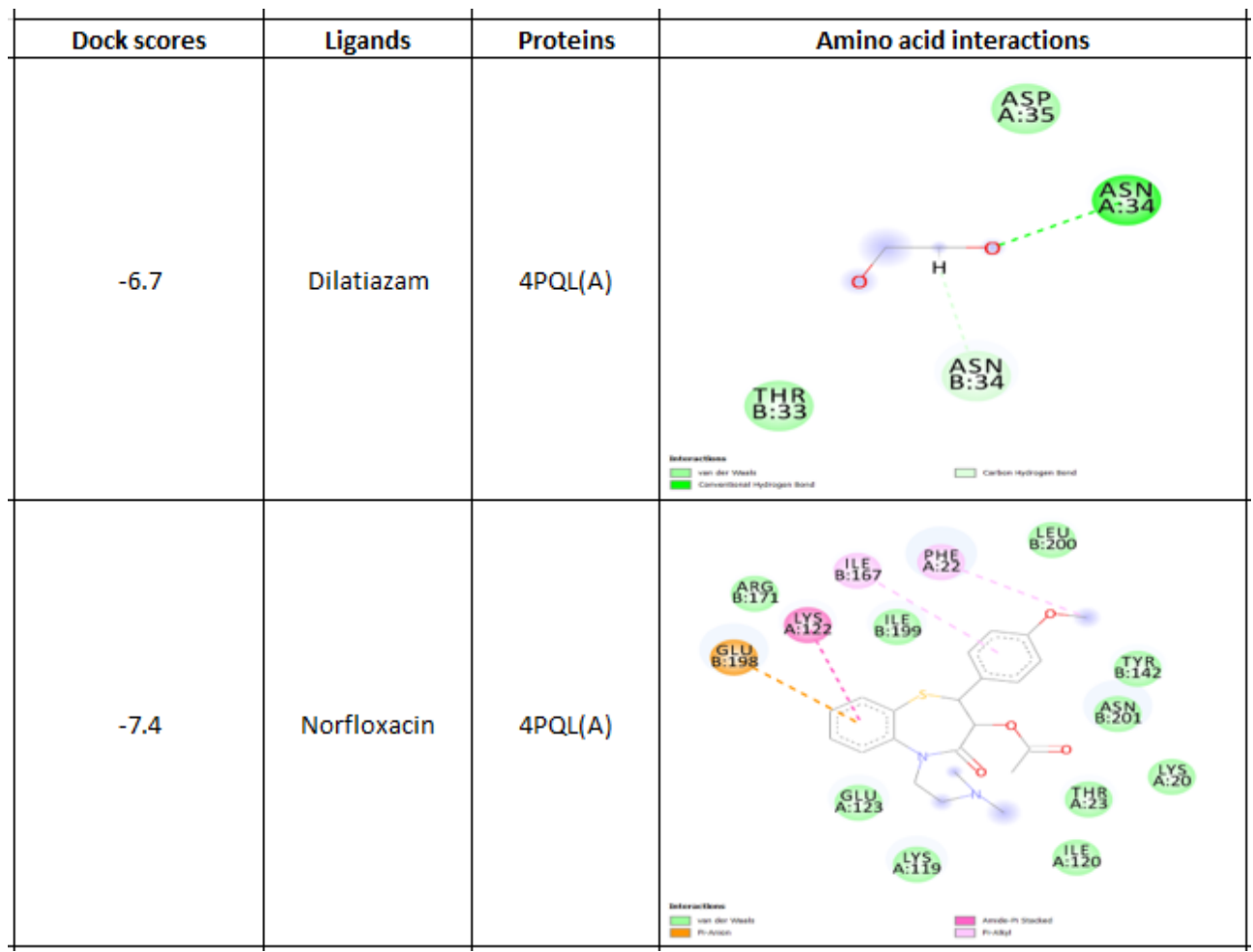


Ranajit Nath et al Int J Sci Res Sci \& Technol. May-June-2021, 8 (3) : 907-919

Table 3.(Contd.) showing interactions and max docking score with -ve sign of Norfloxacin and Ciprofloxacin with protein used in study.

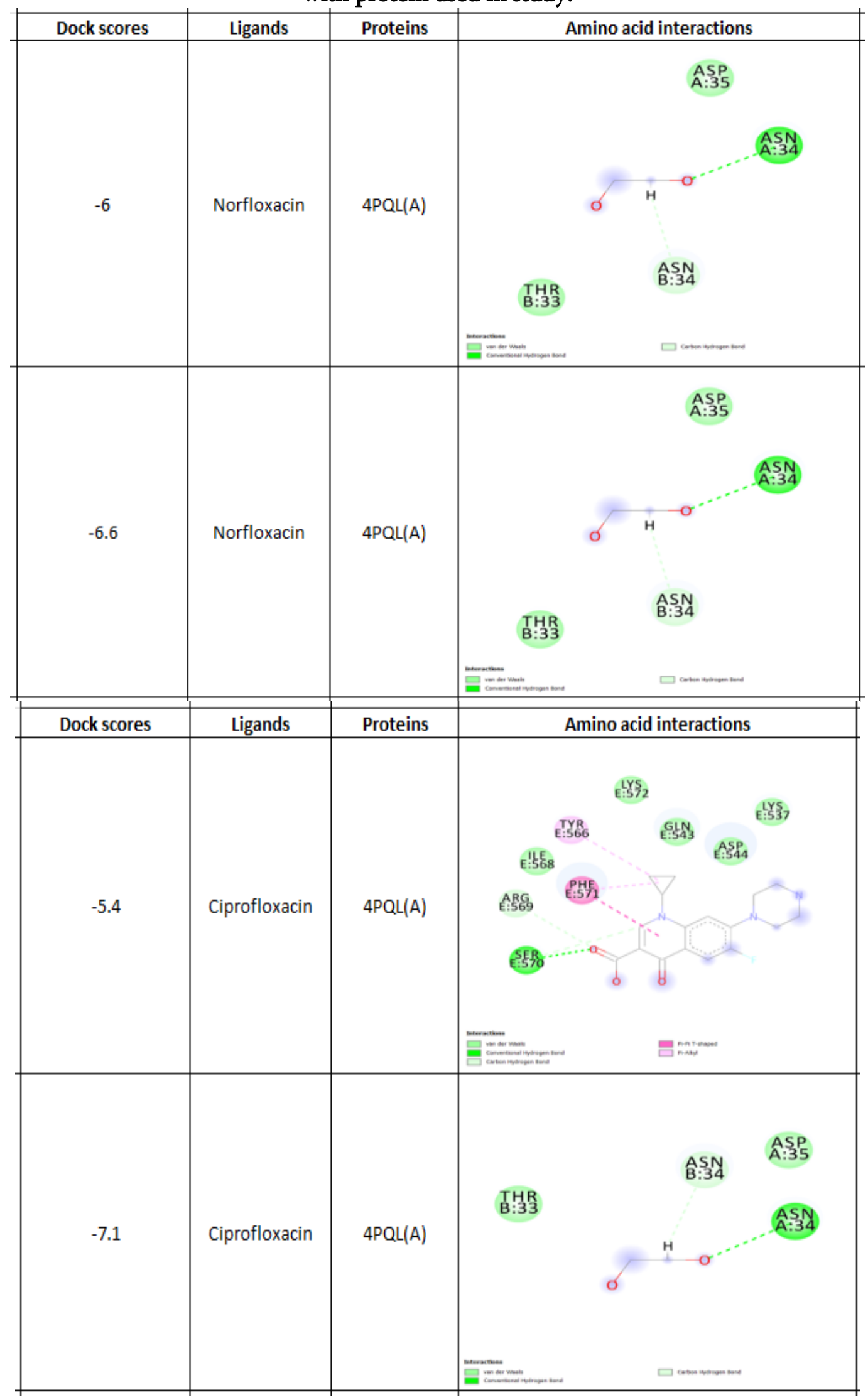


Table 3.(Contd.):showing interactions and max docking score with -ve sign of Moxifloxacin and Ciprofloxacin with protein used in study.

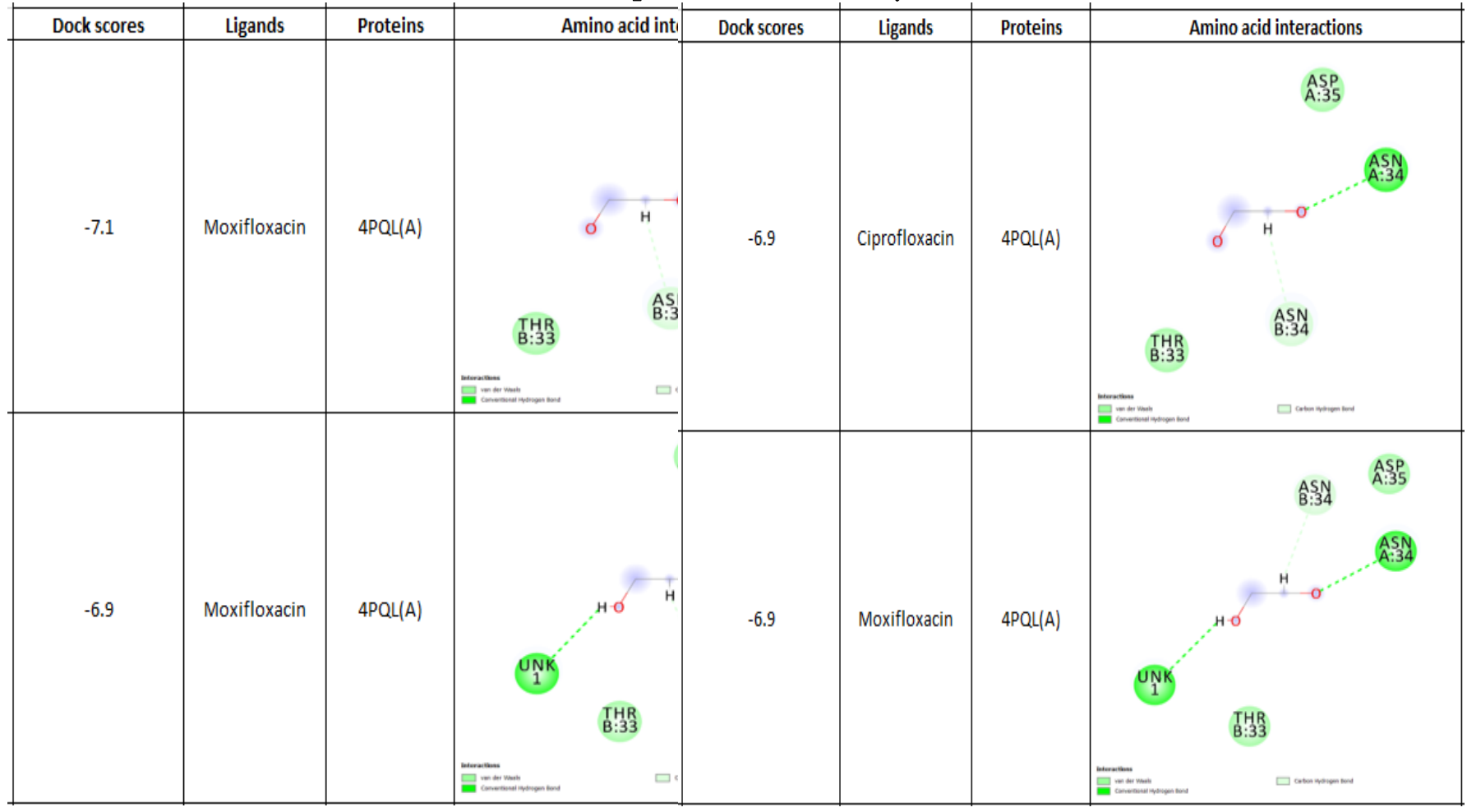

Table 3.(Contd.): showing interactions and max docking score with -ve sign of Moxifloxacin and Ciprofloxacin \& Norffloxacin, Diltiazem with protein used in study.

\begin{tabular}{|c|c|c|c|}
\hline Dock scores & Ligands & Proteins & Amino acid interactions \\
\hline-7 & Norfloxacin & $5 \mathrm{LI} 4(\mathrm{~A})$ & (4F) \\
\hline-8 & Ciprofloxacin & $5 \mathrm{LI} 4(\mathrm{~A})$ & A.4. \\
\hline
\end{tabular}


Ranajit Nath et al Int J Sci Res Sci \& Technol. May-June-2021, 8 (3) : 907-919

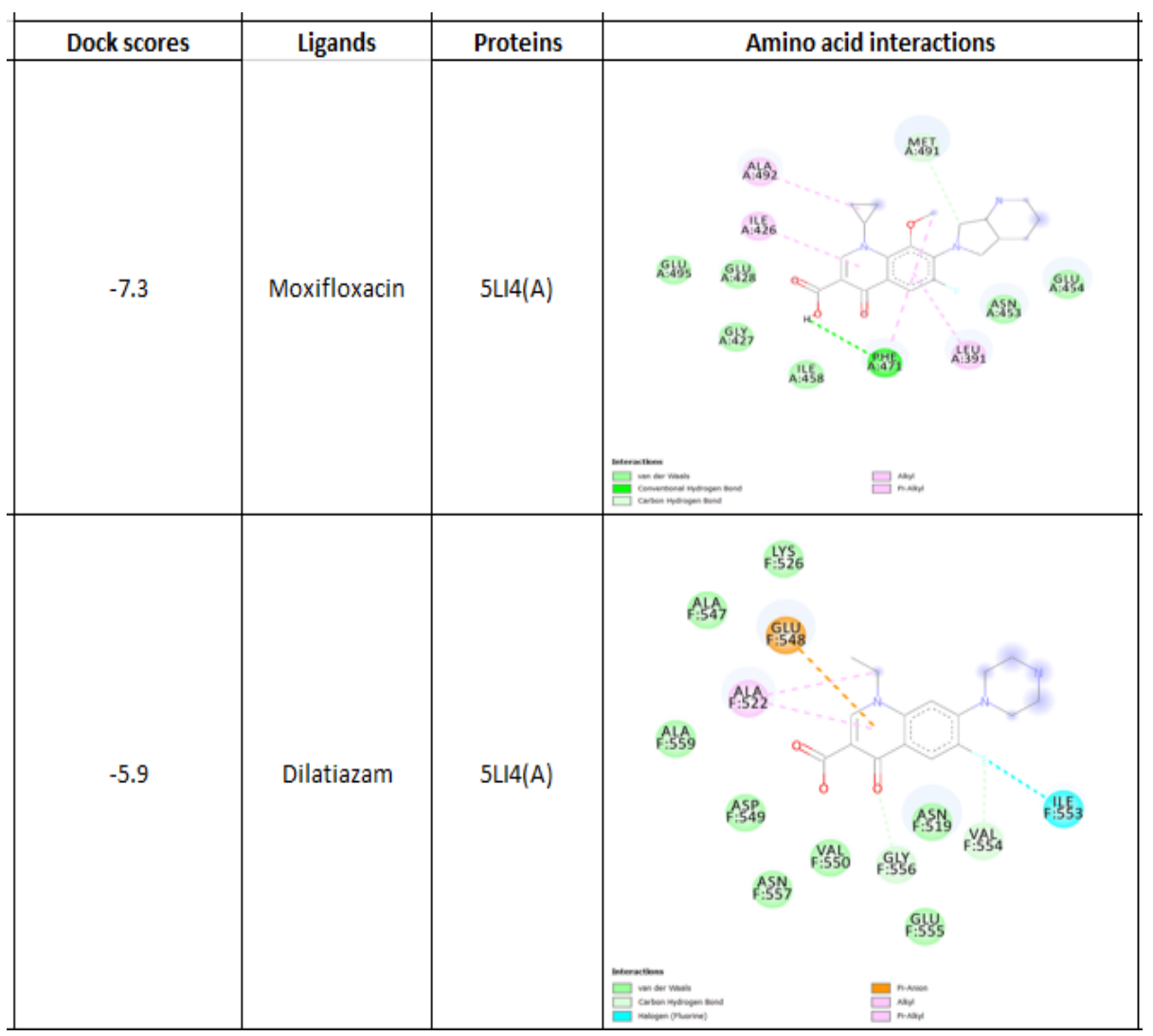

Table 3.(Contd.): showing interactions and max docking score with -ve sign of Moxifloxacin, Ciprofloxacin \& Norffloxacin, Diltiazem with protein used in study

\begin{tabular}{|c|c|c|c|}
\hline Dock scores & Ligands & Proteins & Amino acid interactions \\
\hline-7.7 & Norfloxacin & $5\lfloor\mid 4(A)$ & 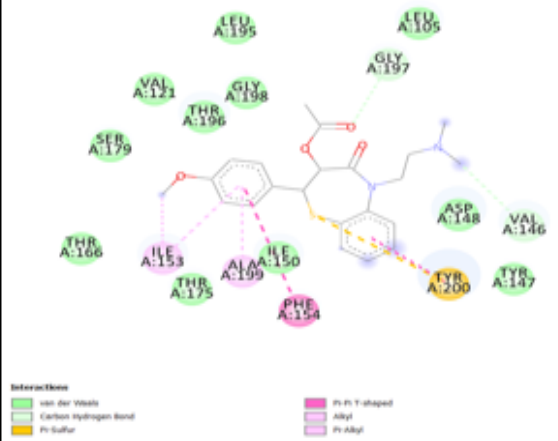 \\
\hline-7.8 & Ciprofloxacin & $5 L 14(\mathrm{~A})$ & (A: \\
\hline
\end{tabular}


Ranajit Nath et al Int J Sci Res Sci \& Technol. May-June-2021, 8 (3) : 907-919

\begin{tabular}{|c|c|c|c|}
\hline Dock scores & Ligands & Proteins & Amino acid interactions \\
\hline-7.4 & Moxifloxacin & $5 L 14(\mathrm{~A})$ & 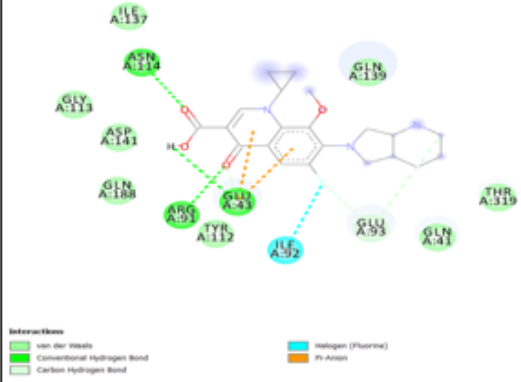 \\
\hline-7.7 & Dilatiazam & $5\llcorner/ 4(A)$ & A AY47 \\
\hline
\end{tabular}

Table 3.(Contd.): showing interactions and max docking score with -ve sign of Moxifloxacin ,Ciprofloxacin \& Norffloxacin, Diltiazem with protein used in study.

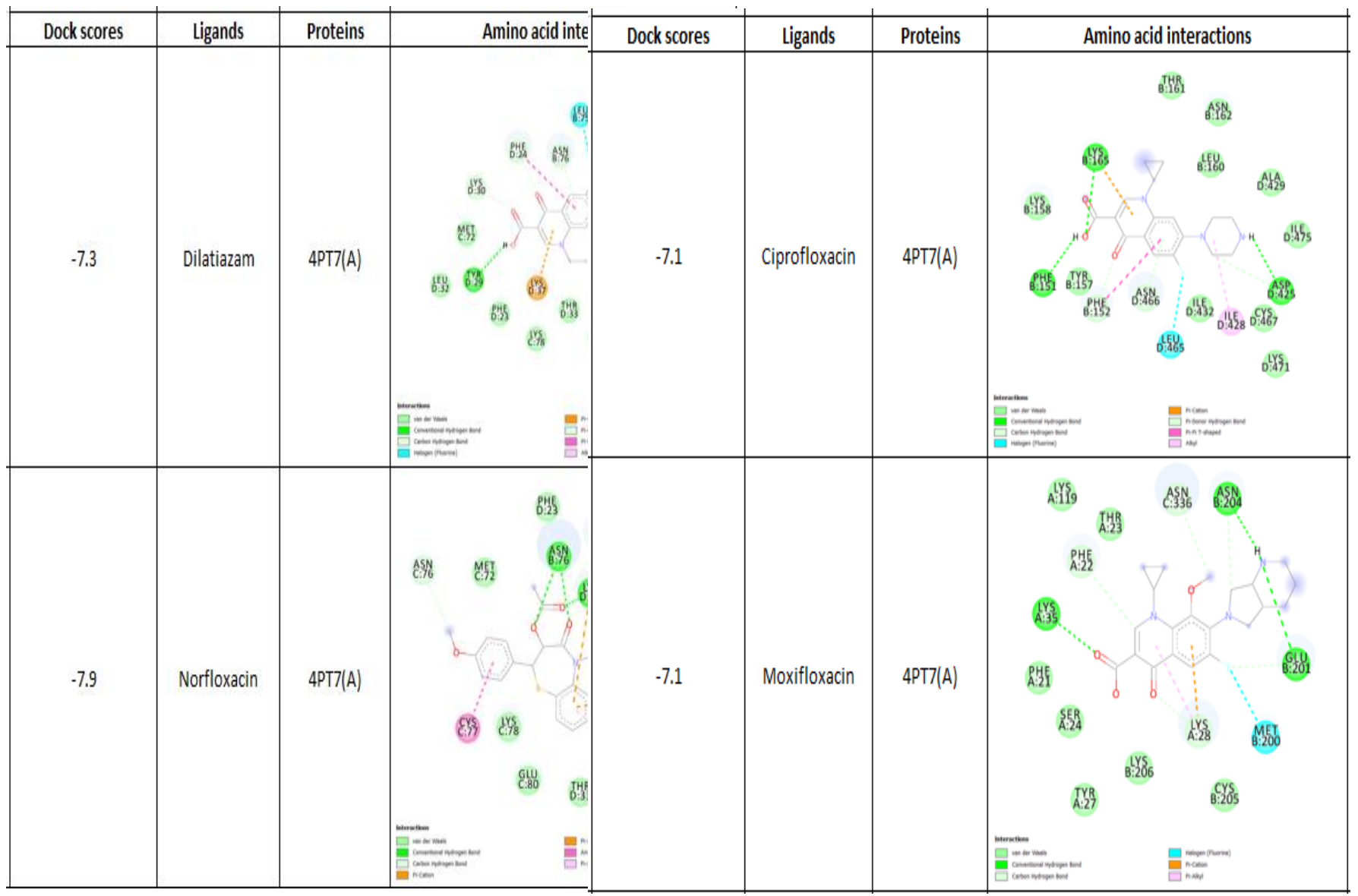


Table 4: shows collective and comparative result of the docking and inhibition shown by ligands to selected proteins based on the above data table and interactions

\begin{tabular}{|l|l|l|l|l|}
\hline LIGANDS & 4PT7(A) & 4PQL(A) & 5LI4(A) & REMARKS \\
\hline CIPROFLOXACIN & YES & YES & YES & HIGH POTENTIAL \\
\hline MOXIFLOXACIN & YES & YES & YES & HIGH POTENTIAL \\
\hline DILTIAZEM & YES & YES & YES & HIGH POTENTIAL \\
\hline NORFLOXACIN & YES & YES & YES & HIGH POTENTIAL \\
\hline
\end{tabular}

\section{IV.CONCLUSION}

Here, all four ligands were studied using bioavailability radar. Our results proposed Moxifloxacin, Ciprofloxacin \& Norffloxacin, Diltiazem showed best docking result Streptococci causing proteins with PDB id's 4PQL, 5LI4 \& 4PT7. To find the effectiveness and to propose the exact mechanism in-vitro studies can be encouraged on Moxifloxacin, Ciprofloxacin \& Norffloxacin, Diltiazem to find a potent cure for the Streptoccocci infection.

\section{REFERENCES}

[1]. Cunningham MW. Post-Streptococcal Autoimmune Sequelae: Rheumatic Fever and Beyond. Streptococcus pyogenes Basic Biol to Clin Manifestations [Internet] 2016;1-37. Available from: http://www.ncbi.nlm.nih.gov/pubmed/2686623 5

[2]. Young PG, Raynes JM, Loh JM, Proft T, Baker EN, Moreland NJ. Group A Streptococcus $t$ antigens have a highly conserved structure concealed under a heterogeneous surface that has implications for vaccine design. Infect Immun 2019;87(6):1-13.

[3]. Stevens DL, Bryant AE. Impetigo, Erysipelas and Cellulitis. Streptococcus pyogenes Basic Biol to Clin Manifestations [Internet] 2016;118. Available from: http://www.ncbi.nlm.nih.gov/pubmed/2686621 1
[4]. Minasyan H. Sepsis: Mechanisms of bacterial injury to the patient 11 Medical and Health Sciences 1108 Medical Microbiology 11 Medical and Health Sciences 1103 Clinical Sciences 06 Biological Sciences 0605 Microbiology. Scand J Trauma Resusc Emerg Med 2019;27(1):1-22.

[5]. Nováček J, Šiborová M, Benešík M, Pantůček R, Doškař J, Plevka P. Structure and genome release of Twort-like Myoviridae phage with a double-layered baseplate. Proc Natl Acad Sci U S A 2016;113(33):9351-6.

[6]. Schumacher MA, Tonthat NK, Kwong SM, Chinnam N babu, Liu MA, Skurray RA, m.fl. Mechanism of staphylococcal multiresistance plasmid replication origin assembly by the RepA protein. Proc Natl Acad Sci [Internet] 2014;111(25):9121-6. Available from: https://www.pnas.org/content/111/25/9121

[7]. Li Y-C, Naveen V, Lin M-G, Hsiao C-D. Structural analyses of the bacterial primosomal protein $\mathrm{DnaB}$ reveal that it is a tetramer and forms a complex with a primosomal reinitiation protein. J Biol Chem [Internet] 2017;292(38):15744-15757. Available from: https://europepmc.org/articles/PMC5612107

[8]. Rusch M, Spielmeyer A, Zorn H, Hamscher G. Biotransformation of ciprofloxacin by Xylaria longipes: structure elucidation and residual antibacterial activity of metabolites. Appl Microbiol Biotechnol 2018;102(19):8573-84.

[9]. Goldstein EJ. Norfloxacin, a fluoroquinolone antibacterial agent. Classification, mechanism of action, and in vitro activity. Am J Med 1987;82(6B):3-17. 
[10]. Xue H, Li M, Liu B, Meng Q. Photochemical degradation kinetics and mechanisms of norfloxacin and oxytetracycline. Environ Sci Pollut Res [Internet] 2021;28(7):8258-65. Available from: https://doi.org/10.1007/s11356020-11181-5

[11]. Guay DR. Moxifloxacin in the treatment of skin and skin structure infections. Ther Clin Risk Manag 2006;2(4):417-34.

[12]. Yeung PK, Prescott C, Haddad C, Montague TJ, McGregor C, Quilliam MA, m.fl. Pharmacokinetics and metabolism of diltiazem in healthy males and females following a single oral dose. Eur J Drug Metab Pharmacokinet 1993;18(2):199-206.

[13]. Daina A, Michielin O, Zoete V. SwissADME: a free web tool to evaluate pharmacokinetics, drug-likeness and medicinal chemistry friendliness of small molecules. Sci Rep 2017;7:42717.

[14]. Berman HM, Kleywegt GJ, Nakamura H, Markley JL. The Protein Data Bank archive as an open data resource. J Comput Aided Mol Des [Internet] 2014;28(10):1009-14. Available from: https://doi.org/10.1007/s10822-014-9770-y

[15]. Berman HM, Westbrook J, Feng Z, Gilliland G, Bhat TN, Weissig H, m.fl. The Protein Data Bank. Nucleic Acids Res [Internet] 2000;28(1):235-42. Available from: https://doi.org/10.1093/nar/28.1.235

[16]. Jiménez J, Doerr S, Martínez-Rosell G, Rose AS, De Fabritiis G. DeepSite: protein-binding site predictor using 3D-convolutional neural networks. Bioinformatics 2017;33(19):3036-42.

[17]. Pettersen EF, Goddard TD, Huang CC, Couch GS, Greenblatt DM, Meng EC, m.fl. UCSF Chimera - A visualization system for exploratory research and analysis. J Comput Chem 2004;25(13):1605-12.

[18]. Trott O, Olson AJ. AutoDock Vina: Improving the speed and accuracy of docking with a new scoring function, efficient optimization, and multithreading. J Comput Chem 2009;31(2):NANA.

[19]. L DeLano W. Pymol: An open-source molecular graphics tool. \{CCP4\} Newsl Protein Crystallogr [Internet] 2002;40:1-8. Available from: http://www.ccp4.ac.uk/newsletters/newsletter4 0/11_pymol.pdf

[20]. Kemmish H, Fasnacht M, Yan L. Fully automated antibody structure prediction using BIOVIA tools: Validation study. PLoS One 2017;12(5):1-26.

[21]. Love J, Selker R, Marsman M, Jamil T, Dropmann D, Verhagen J, m.fl. JASP: Graphical statistical software for common statistical designs. J Stat Softw 2019;88(1).

\section{Cite this article as :}

Ranajit Nath, Ratna Roy, Soubhik bhattacharyya, Sourav Datta, " Drug Repurposing: In Silico Modeling of Streptococcus Infection", International Journal of Scientific Research in Science and Technology(IJSRST), Print ISSN : 2395-6011, Online ISSN : 2395-602X, Volume 8, Issue 3, pp.907-919, May-June-2021. Available at doi : https://doi.org/10.32628/IJSRST2183191 Journal URL : https://ijsrst.com/IJSRST2183191 
Ranajit Nath et al Int J Sci Res Sci \& Technol. May-June-2021, 8 (3) : 907-919 